\title{
Design of Cationic Multi-Walled Carbon Nanotubes as Efficient siRNA Vectors for Lung Cancer Xenograft Eradication
}

Chang Guo, Wafa T Al-Jamal, Francesca M Toma, Alberto Bianco,

Maurizio Prato, Khuloud T Al-Jamal, and Kostas Kostarelos

Bioconjugate Chem., Just Accepted Manuscript • DOI: 10.1021/acs.bioconjchem.5b00249 • Publication Date (Web): 03 Jun 2015

Downloaded from http://pubs.acs.org on June 15, 2015

Just Accepted

"Just Accepted" manuscripts have been peer-reviewed and accepted for publication. They are posted online prior to technical editing, formatting for publication and author proofing. The American Chemical Society provides "Just Accepted" as a free service to the research community to expedite the dissemination of scientific material as soon as possible after acceptance. "Just Accepted" manuscripts appear in full in PDF format accompanied by an HTML abstract. "Just Accepted" manuscripts have been fully peer reviewed, but should not be considered the official version of record. They are accessible to all readers and citable by the Digital Object Identifier (DOI®). "Just Accepted" is an optional service offered to authors. Therefore, the "Just Accepted" Web site may not include all articles that will be published in the journal. After a manuscript is technically edited and formatted, it will be removed from the "Just Accepted" Web site and published as an ASAP article. Note that technical editing may introduce minor changes to the manuscript text and/or graphics which could affect content, and all legal disclaimers and ethical guidelines that apply to the journal pertain. ACS cannot be held responsible for errors or consequences arising from the use of information contained in these "Just Accepted" manuscripts.

\section{ACS Publications}




\section{Design of Cationic Multi-Walled Carbon Nanotubes as Efficient siRNA} Vectors for Lung Cancer Xenograft Eradication

Chang Guo ${ }^{a}$, Wafa T. Al-Jamal ${ }^{a}$, Francesca M. Toma ${ }^{b}$, Alberto Bianco $^{c}$, Maurizio Prato ${ }^{b}$, Khuloud T. AlJamal ${ }^{\mu^{\prime}, *}$ and Kostas Kostarelos ${ }^{a}$

${ }^{a}$ Nanomedicine Lab, UCL School of Pharmacy, Faculty of Life Sciences, University College London, London WC1N 1AX, UK

${ }^{b}$ Center of Excellence for Nanostructured Materials, Department of Pharmaceutical Sciences, University of Trieste, 34127 Trieste, Italy ${ }^{\circ}$ CNRS, Institut de Biologie Moléculaire et Cellulaire, UPR 9021 Immunologie et Chimie Thérapeutiques, 67000 Strasbourg, France

* To whom correspondence should be addressed to either: khuloud.al-jamal@kcl.ac.uk; k.kostarelos@ucl.ac.uk

${ }^{\wedge}$ Current address: Institute of Pharmaceutical Sciences, King's College London, Franklin-Wilki1kns Building, London SE1 $9 \mathrm{NH}, \mathrm{UK}$

ACS Paragon Plus Environment 


\section{ABSTRACT}

Polo-Like Kinase (PLK1) has been identified as a potential target in cancer gene therapy via chemical or genetic inhibitory approaches. The biomedical applications of chemically functionalized carbon nanotubes ( $f$-CNTs) in cancer therapy have been studied due to their ability to efficiently deliver siRNA intracellularly. In this study, we established the capacity of cationic MWNT- $\mathrm{NH}_{3}{ }^{+}$to deliver the apoptotic siRNA against PLK1 (siPLK1) in Calu6 tumor xenografts by direct intratumoural injections. A direct comparison with cationic liposomes was made. This study validates the PLK1 gene as a potential target in cancer gene therapy including lung cancer, as demonstrated by the therapeutic efficacy of siPLK1:MWNT- $\mathrm{NH}_{3}{ }^{+}$complexes and their ability to significantly improve animal survival. Biological analysis of the siPLK1:MWNT- $\mathrm{NH}_{3}{ }^{+}$treated tumors by RT-PCR and Western blot, in addition to TUNEL staining confirmed the biological functionality of the siRNA intratumorally, suggesting that tumor eradication was due to PLK1 knockdown. Furthermore, by using a fluorescently labeled, non-coding siRNA sequence complexed with MWNT- $\mathrm{NH}_{3}{ }^{+}$, we established for the first time that the improved therapeutic efficacy observed in $f$ CNT-based siRNA delivery is directly proportional to the enhanced siRNA retention in the solid tumor and subsequent uptake by tumor cells after local administration in vivo. 


\section{INTRODUCTION}

Over the past decade, there has been an exponential growth in research combining nanotechnology tools and cancer therapy. One of nanomaterials are carbon nanotubes (CNTs) which based on their structure are classified into two categories, single-walled carbon nanotubes (SWNTs) and multi-walled carbon nanotubes (MWNTs) made of single or several graphene layers rolled up into cylindrical structures, respectively. The capability of surface modification of CNTs offers an improved aqueous dispersibility hence broadening their biomedical applications, including their use as a delivery vector for various molecules in cancer therapy. Functionalized CNT ( $f$-CNT) have been explored in almost every single modality used in cancer therapy including photothermal therapy, chemotherapy and gene therapy. ${ }^{1}$

In comparison to traditional chemotherapy or radiotherapy, gene therapy offers higher specificity to regulate diseases of disordered genes origin, including cancer. The induction of apoptosis has demonstrated great benefits for cancer therapy. ${ }^{2,3}$ One of the cancer-related genes, polo-like kinase 1 (PLK1) has been attracting increased attention in terms of cancer therapy. These highly conserved serine/threonine kinases are the principal kinase proteins which serve as regulatory enzymes for mitotic events by performing phosphorylation at nuclear protein B23. ${ }^{4,5}$ They play an important role in G2-M transition and show an over-expression in a variety of tumors analyzed to date. ${ }^{6,7}$ Therefore, inhibition of PLK1 is predicted to block the reproduction of cancer cells, thus indicating its potential as a promising cancer drug target. Studies carried out so far have been exploring both chemical and genetic inhibition approaches. A chemical approach using a stilbazole compound, $\mathrm{HMN}-176$, and its orally bioavailable prodrug $\mathrm{HMN}-214$, have both shown potent cytotoxic activity against several tumor cell lines. ${ }^{8}$ Garland and co-workers believe that these compounds interact with PLK1 by interference with its normal subcellular spatial distribution at centrosomes and along the cytoskeletal structure, but not via direct inhibition. An alternative genetic approach whereby silencing PLK1 by siRNA induces apoptosis via inactivation of cyclindependent mitotic arrest has already shown some promising results. ${ }^{9,10}$ Strebhardt's group has revealed an elevated number of apoptotic nuclei, an increased sub-2N DNA content, and up to 5fold increases in G2-M compared with controls, followed by apoptosis after depletion of PLK1 in cancer cells. ${ }^{11,12}$ Subsequent studies combining PLK1 gene silencing with chemotherapeutics such as paclitaxel, carboplatin or Herceptin showed improved sensitivity of breast cancer cells against drug therapy both in vitro and in vivo. ${ }^{13,14}$

$f$-CNTs have been employed as non-viral gene delivery vectors offering several advantages compared to alternative non-viral vectors including liposomes, cationic polymers and cellpenetrating peptides. Advantages include efficient membrane translocation and in vivo stability. ${ }^{15,16}$ $f$-CNTs are able to deliver nucleic acids such as plasmid DNA, siRNA or aptamers intracellularly. In these studies, siRNA:f-CNT complexation was achieved by (i) chemical conjugation of siRNA to the vector via cleavable disulfide linkage, (ii) adsorption of siRNA on the PEG-phospholipid coat adsorbed onto the SWNTs, or (iii) by non-covalent complextion between the negatively charged siRNA and the cationic $f$-CNTs. Effective gene silencing was observed in all of these studies thereby indicating the potential of CNT-based delivery of siRNA in mammalian cells. Furthermore, both SWNTs ${ }^{17-21}$ and MWNTs ${ }^{22-24}$ have shown promise as siRNA transporters in vitro and in vivo in the field of cancer therapy. In a study performed by Wang et al., $f$-CNT-based transfection of cyclinA siRNA was found to successfully suppress cyclinA expression, resulting in cell growth inhibition and apoptosis induction. ${ }^{19}$ Moreover, several reports have shown the therapeutic effect and suppression of tumor growth when $f$-CNTs were employed as siRNA delivery systems in vivo. ${ }^{18,22,25}$ Bartholomeusz et al., observed biological effects after exposing a wide variety of 
cancer cell types in vitro to siRNA:SWNT complexes. The same study showed that intratumoral administration of HIF-1 $\alpha$ siRNA:SWNT complexes resulted in inhibition of HIF-1 $\alpha$ activity in a xenograft mouse tumor model. ${ }^{25}$ Furthermore, another study demonstrated that when positively charged SWNT-CO $\left(\mathrm{CH}_{2}\right)_{6} \mathrm{NH}_{3}{ }^{+}$are employed for siRNA delivery, suppression of telomerase reverse transcriptase (TERT) expression is observed both in vitro and in vivo, resulting in inhibition of the tumor growth in both murine and human tumor models. ${ }^{18}$ We have also reported antitumor activity in the human lung carcinoma (Calu6) xenograft model and prolonged animal survival by delivering a proprietary toxic siRNA sequence (siTOX using amino-functionalized multi-walled carbon nanotubes (MWNT-NH${ }_{3}^{+}$), ${ }^{22}$ which has been also utilized in this study.

Herein, we validated PLK1 as a target in cancer gene therapy and demonstrated by combining PLK1 siRNA (siPLK1, a siRNA sequence specific for PLK1) with nanotechnology tools ( $f$-CNT delivery vectors) an improved therapeutic efficacy and observed prolonged animal survival Moreover, we explored for the first time the correlation between siRNA retention within the solid tumor together with their cellular uptake in tumor cells in vivo and the resultant therapeutic outcome. Improved tumor retention and enhanced cellular uptake of siRNA:MWNT- $\mathrm{NH}_{3}{ }^{+}$ complexes appear to play an essential role in improving the therapeutic outcome after local administration of siRNA in vivo.

\section{RESULTS}

siPLK1 expression in siPLK:f-MWNT complexes treated group. The chemical structure of $f$ MWNT (MWNT-NH ${ }_{3}^{+}$) studied here is shown in Fig. S1. To validate the PLK1 siRNA (siPLK1) sequence in vitro, Calu6 cells were incubated with siPLK1:f-MWNT complexes (1:8 mass ratio) for 24 or 48 hours. The level of PLK1 expression in Calu6 cells was assessed by real-time PCR and Western blotting (Fig. 1A, B \&C). GAPDH was used as a housekeeping gene for real-time PCR and as a control for Western blotting. Lipoplexes formed by complexation of SiPLK1 and cationic liposomes (DOTAP:cholesterol) were included in the study as a benchmark transfecting reagent. There was a clear difference in gene silencing efficacy between the two vectors in vitro, where lipoplexes exhibited more efficient PLK1 gene knockdown than siPLK1:f-MWNT complexes as measured by PLK1 protein level (Fig. 1A\&B). Protein expression was reduced to $30 \% \pm 1 \%$ and $20 \% \pm 2 \%$ after 24 and 48 hours, respectively, in case of the lipoplex transfection compared to only $66 \% \pm 3 \%$ and $63 \% \pm 4 \%$ in case of transfection using siPLK1:f-MWNT complexes after the same incubation times (Fig. 1B). Figure 1C shows that incubation of Calu6 cells with siRNA:f-MWNT complexes decreased the number of mRNA copies of the PLK1 to $66 \% \pm 9 \%$ and $68 \% \pm 4 \%$ at 6 and 24 hours, respectively, as shown by real-time PCR. In comparison, incubation of Calu6 cells with lipoplexes attenuated relative PLK1 mRNA level to $22 \% \pm 1 \%$ and $25 \% \pm 1 \%$ at 6 and 24 hours, respectively. Hence, we concluded that more potent gene silencing could be achieved using the lipoplexes than siRNA:f-MWNT complexes in vitro.

We then investigated if siPLK1 is still active under biological conditions in vivo. SiPLK1 was complexed with either $f$-MWNTs or cationic liposomes and directly introduced into Calu6 tumors by intratumoral injection. PLK1 mRNA transcripts and protein were extracted from injected tumors and PLK1 expression was evaluated by real-time PCR and Western blotting (Fig. 1D, E\&F) in all groups. Negative siRNA siNEG:f-MWNTs treated group was included to eliminate any non-specific toxicity of the siRNA or the $f$-MWNTs. Both techniques demonstrated a significant reduction in PLK1 mRNA transcripts and protein levels in the tumors treated with siPLK1:f-MWNT complexes 
compared to the injected tumours of control groups ( $5 \%$ dextrose dextrose and siPLK1 alone) tumors. Intratumoral injection of siPLK1:f-MWNT complexes reduced PLK1 mRNA levels to $67 \% \pm$ $6 \%$ and PLK1 protein levels to $38 \% \pm 8 \%$ relative to expression of the housekeeping gene GAPDH (Fig. 1D, E\&F). Lipoplexes and siNEG: $f$-MWNT treated groups exhibited different effects in PLK1 silencing both in vitro and in vivo. Such results proposed that siPLK1:f-MWNT complexes as promising vectors for RNAi in vivo. Furthermore, the delivery capacity of well-established delivery vectors such as liposomes which have shown to be potent in vitro can be compromised when administered locally within tumor environment in vivo.

A

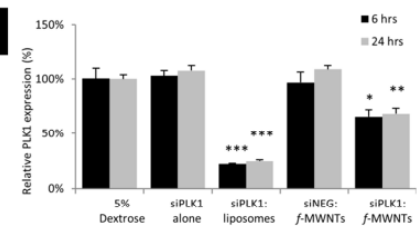

B

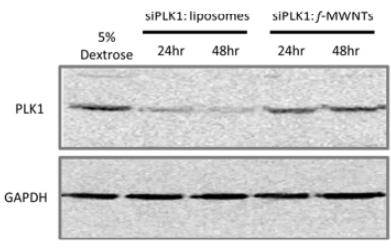

c

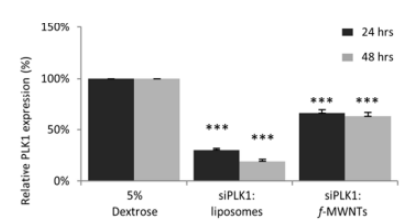

D

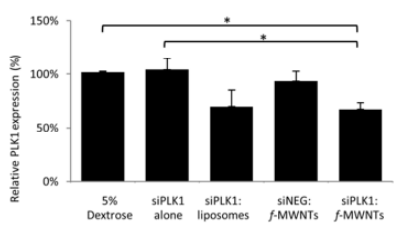

E

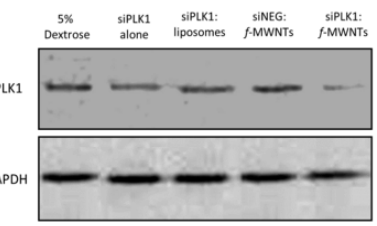

$\mathbf{F}$

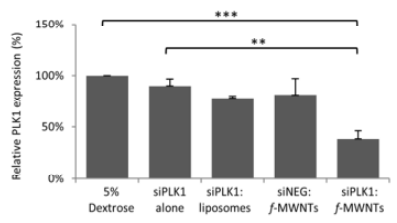

Figure 1: PLK1 gene silencing analysis in Calu6 cell lines and tumor xenofrafts. (A-C) In vitro gene silencing. Calu6 monolayers were treated with siPLK1 at $100 \mathrm{nM}$ final concentration in serum free media for 4 hours followed by incubation in complete media for another 6 hours, 24 hours or 48 hours. GADPH gene was used as a control housekeeping gene. Treatment groups include 5\% dextrose, siPLK1 alone, lipoplexes (siPLK1 in complex with
DOTAP:cholesterol liposomes, (4:1 N/P)), siNEG:f-MWNT complexes (1:8 mass ratio) and siPLK1:f-MWNT complexes (1:8 mass ratio). (A) mRNA expression levels of PLK1 analyzed in Calu6 monolayers lysates by real time PCR $(n=3)$ at 6 hours or 24 hours post-incubation. (B\&C) Western blots of cell lysates to determine PLK1 expression at 24 hours or 48 hours post-incubation. Both protein and mRNA levels of PLK1 were reduced more efficiently in case of lipoplexes compared to siPLK1:f-MWNT complexes. Results were analyzed using $t$-test between treated groups and control groups ( $5 \%$ Dextrose) at different time points respectively. (D-F) In vivo gene silencing. Calu6 xenograft tumors were administered with siPLK1 at a dose of $4 \mu \mathrm{g}$ per tumor in $5 \%$ dextrose on days 17, 20,23, 27 and 30 . GADPH gene was used as a control housekeeping gene. Treatment groups include $5 \%$ dextrose, siPLK1 alone, lipoplexes, siNEG:f-MWNT complexes and siPLK1:f-MWNT complexes. (D) mRNA expression levels of PLK1 analyzed in Calu6 tumor lysates by real time PCR $(n=3)$ post-administration. (E\&F) Western blots of cell lysates to determine PLK1 expression in the lysates. Both protein and mRNA levels of PLK1 were reduced more efficiently in case of siPLK1: $f$-MWNT complexes compared to lipoplexes. Results were analyzed using $t$-test between group of siPLK1:f-MWNT complexes and other groups ( $p$ values $={ }^{*}<0.05 ;{ }^{* *}<0.01 ;{ }^{* * *}<0.001$ ). 
Tumor growth and animal survival in vivo after intratumoral administration of siPLK1:fMWNT complexes. We further studied if gene silencing by siPLK:f-MWNT complexes is sufficient to delay tumor growth and improve animal survival. For this purpose, treatments were injected intratumorally in an established human lung Calu6 xenograft tumor model. Tumor growth was monitored by measuring the tumor size three times per week with Vernier calipers over a period of 30 days. Figure 2A showed a significant inhibition in tumor growth observed in siPLK1:f-MWNT complexes treated group. Reductions in tumor volume were noticeable after the second therapeutic injection. Other treatment groups (siPLK1 alone, siPLK1:liposomes, siNEG:f-MWNT complexes) showed no difference in volume compared to the $5 \%$ dextrose treated group. To compare the efficiency of different types of therapies used, quadrupling time was calculated and expressed in Figure 2B. Quadrupling time of siPLK1:f-MWNTs treated group was $20.5 \pm 2.3$ days $(n=9)$ compared to only $11.3 \pm 0.4$ days $(n=10)$ in the case of $5 \%$ dextrose treated group. siPLK1:fMWNT group exhibited significant increase in quadrupling time compared to all other treated groups. Animal survival analysis is shown in Fig. 2C. siPLK1:f-MWNT complexes treated group showed significant increase in animal survival compared to all other groups ( $5 \%$ dextrose, siPLK1 alone, siPLK1:liposomes, siNEG:f-MWNT complexes). There was no significant improvement in animal survival for those treated with siPLK1:liposomes compared to $5 \%$ dextrose or siPLK1 alone.

\section{A}

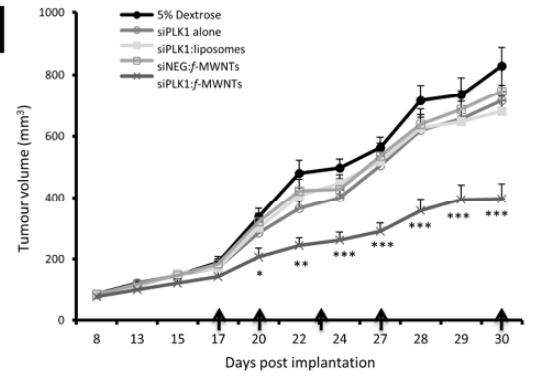

B

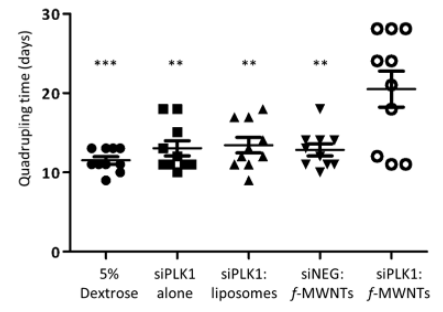

c

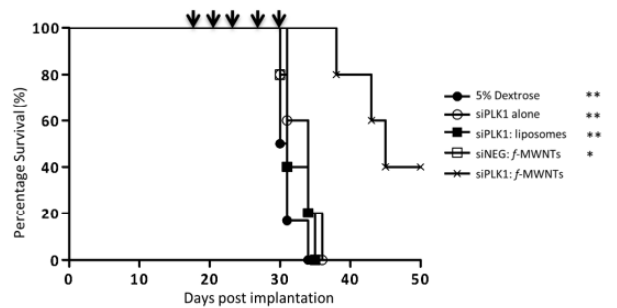

Figure 2: Tumor growth and survival curves in Calu6 xenografts bearing mice. (A) Growth curves analysis of Calu6 xenografts. Female Swiss nude mice Calu6 xenograft tumors were administered with siPLK1. Treatment groups include siPLK1 (4 $\mu \mathrm{g})$ in complex with either $f$-MWNTs $(1: 8$ mass ratio) or DOTAP:cholesterol liposomes (N/P=1/4; $4 \mu \mathrm{g}$ siRNA). siNEG:f-MWNT (1:8 mass ratio) complexes and siPLK1 alon were injected as negative controls. Arrowheads indicate injections on days 17 , 20, 2327 and 30. Results were presented as mean $+/$ - SEM $(n=9-10)$. (B) Tumor volume quadrupling times (TVQT, in days) for treatment and control groups. (C) Survival analysis of Calu6 xenograft bearing mice. Formulations: $5 \%$ dextrose as control (circle); siPLK1 alone $(4 \mu \mathrm{g}$ ) (open circle); lipoplexes (N/P=1/4; $4 \mu \mathrm{g}$ siRNA) (square); siNEG: $f$-MWNT complexes (mass ratio $=1: 8$; $4 \mu \mathrm{g}$ siRNA) (open square); siPLK1:f-MWNT complexes (mass ratio $=1: 8 ; 4 \mu \mathrm{g}$ siRNA) (star). Results were analyzed using $t$-test between group of siPLK1:f-MWNT complexes and other groups $(\mathrm{p}$ values $=$ ${ }^{*}<0.05 ;^{* *}<0.01 ;^{* * *}<0.001$ ).

ACS Paragon Plus Environment 
Observation of siPLK1:f-MWNT complexes in tumor mass and the toxicity of $f$-MWNT vehicle to tumor cells. Tumors were excised when the tumors reached their maximum growth volume allowed by the UK Home Office. Figure 3 shows a representative cross section image of the whole tumor from each treatment group. The presence of $f$-MWNT in siNEG: $f$-MWNTs and siPLK1:f-MWNTs treated tumors was confirmed by the greyish color in the core of these tumors (at 100X magnification). Tumors were fixed in formalin and processed for either neutral red or H\&E staining in order to perform histological examination of the tumors at the microscopic level. Neutral red staining (at 400X magnification) confirmed the presence of $f$-MWNTs in tumor cells. Moreover, $\mathrm{H} \& \mathrm{E}$ staining revealed extended necrosis in the areas around the CNTs only in the siPLK1:fMWNT treated group. Tumors treated with siNEG:f-MWNT complexes lacked areas of necrotic tissues that were apparent in siPLK1:f-MWNTs group, indicating that the toxicity seen was due to siPLK silencing after being internalized into the cells. Major organs such as heart, spleen, lung, liver and kidney were also dissected and sectioned for neutral red and H\&E staining (Supplementary Fig. S2). However, $f$-MWNTs could not be detected in these organs indicating there was retention of the vectors in tumor tissue after intratumoral injection.

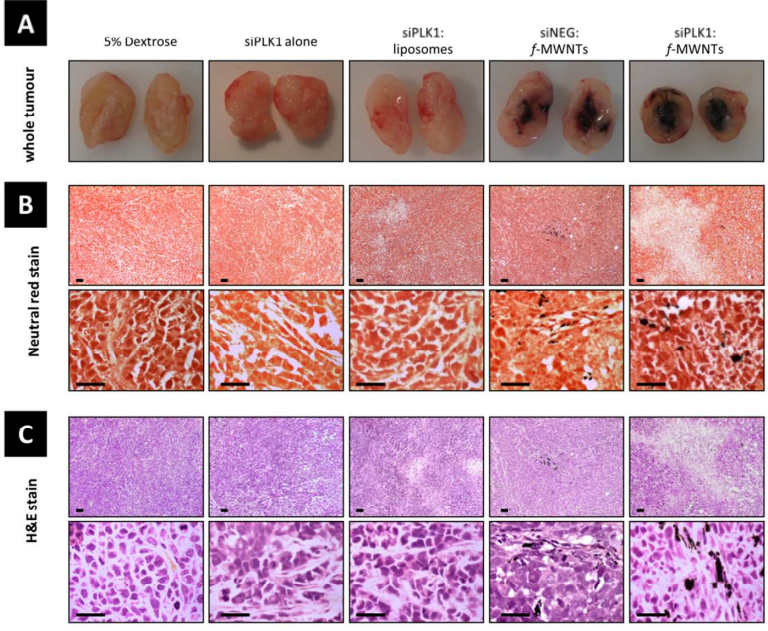

Figure 3: Histological analysis of Calu6 tumor xenografts. (A) Photographs of whole tumors excized once reaching $800 \mathrm{~mm}^{3}$ volume. Histological examination of fixed tumor sections processed for routine histology with (B) neutral red stain or (C) and hematoxylin and eosin (H\&E) stain. Tumors treated with siPLK1:f-MWNT complexes showed significant tissue necrosis compared to all other treated groups.

Apoptosis induction in tumor mass by PLK1 silencing. To confirm if apoptosis was the mechanism of cell death as a result of PLK1 silencing, TUNEL staining was performed and all nuclei were counterstained by using DAPI stain (Fig. 4). Only apoptotic cells exhibited green fluorescence (TUNEL positive). siPLK1:f-MWNT treated tumors were TUNEL positive in the same regions where $f$-MWNTs were abundant as confirmed by DIC images. Similar tumor regions exposed to siNEG:f-MWNT complexes were TUNEL negative. This result further confirms that apoptosis may be attributable to cell death induced by PLK1 gene silencing. The groups treated with $5 \%$ dextrose and lipoplexes showed predominantly healthy appearance with scarce areas of TUNEL positive cells which could be due to a natural process of cell death during tumor growth. 


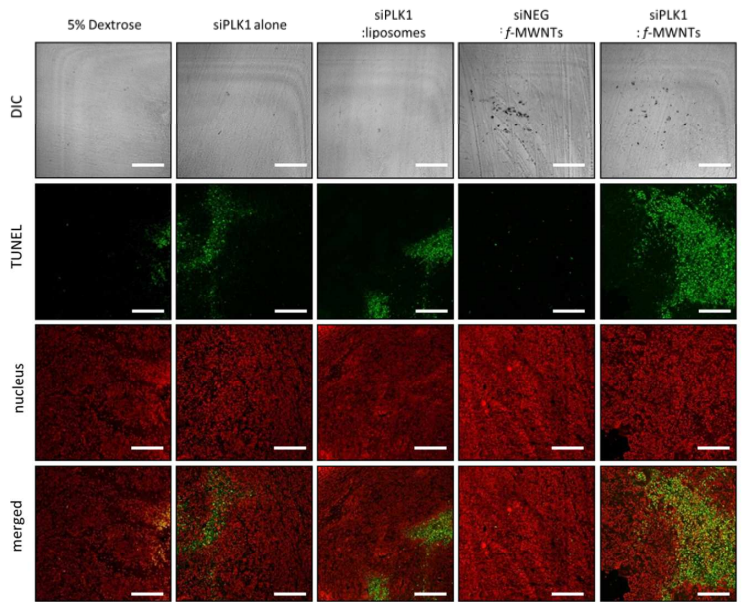

Figure 4: TUNEL analysis of Calu6 tumor xenografts. Tumor sections were deparaffinised and rehydrated through graded ethanol then TUNEL stained to identify apoptotic (green) cells from counterstained nuclei (red). Phase contrast images of corresponding fields of view shown to indicate $\mathrm{MWNT}-\mathrm{NH}_{3}{ }^{+}$localization in tumor tissues. Tumors treated with siPLK1:f-MWNT complexes showed significant apoptosis of the tumor cells. Scale bar is $200 \mu \mathrm{m}$

siRNA retention in tumor mass after local administration of siRNA: $f$-MWNT complexes. In order to investigate the kinetics of siRNA clearance from the tumor mass and more importantly to learn about the interaction between siRNA and tumor cells, we have substituted the siPLK1 with fluorescently labeled negative siRNA with AlexaFluor AF546 (siNEG AF546). Whole body imaging in addition to imaging of excised tumors (ex vivo) was carried out after single intratumoural injections (Supplementary Fig. S3 and Fig. 5) using the Xenogen IVIS imaging system to visualize the fluorescence signal representing siNEG AF546 complexed with both f-MWNT and liposomes. It was clear from whole body imaging that the lipoplex group exhibited the long-lasting retention in the tumor mass up to $24 \mathrm{~h}(47.5 \%$ ID $\pm 3.0 \%$ ID after $1 \mathrm{~h}$ and $21.3 \%$ ID $\pm 4.6 \%$ ID after $4 \mathrm{~h}, \mathrm{n}=6)$. On the contrary, siNEG AF546 alone cleared rapidly from the tumor mass with $26.6 \%$ ID $\pm 3.8 \% \mathrm{ID}(\mathrm{n}=6)$ and $8.9 \% \mathrm{ID} \pm 2.0 \%$ ID detected in the tumors after 1 and $4 \mathrm{~h}$, respectively (Supplementary Fig. S3). Interestingly, the siNEG AF546:f-MWNT treated group exhibited lower signals compared to the lipoplex formulation by whole body imaging technique, with $62.3 \%$ ID \pm $6.3 \%$ ID and $15.2 \%$ ID $\pm 1.5 \%$ ID $(n=6)$ remaining within the tumor mass at 1 and $4 \mathrm{~h}$ post-injection, respectively.

Imaging of tumors (ex vivo) was then performed to account for problems associated with deep tissue penetration of fluorescence signals (Fig. 5). Tumors were dissected 24 hours after intratumoral administration of free or complexed siNEG AF546 and observed under Xenogen IVIS imaging system (Figure 5A). Similar tumor retention profiles were obtained ex vivo as those obtained by whole body imaging. To confirm whether the fluorescence detected by IVIS was associated with tumor cells or within the interstitial space, tumor masses were excised, cryosectioned, post-fixed, and examined by confocal laser scanning microscopy (CLSM) (Figure 5B). During the fixation and subsequent washing steps, free non-associated siNEG AF546 was thus 
eliminated before imaging by CLSM. At $24 \mathrm{~h}$ post-intratumoral administration, siNEG AF546 treated group showed no fluorescence signals indicating that siRNA alone was not able to internalize into cancer cells without the aid of a delivery system. siNEG AF546:f-MWNT complexes treated tumors showed intense red fluorescence signals confirming intracellular delivery of siNEG AF546 by means of $f$-MWNTs. Moreover, fluorescence signals in the latter group were extended throughout the cells including the nucleus. Such results were compatible with the results we previously obtained in cancerous cell monolayer studies. ${ }^{26,27}$ Surprisingly and contrary to whole tumor imaging studies (Figure 5A), intracellular delivery of siNEG AF546 by lipoplexes appeared to be less intense than the $f$-MWNT-mediated delivery. Such results were in line with the reduced gene silencing efficiency obtained in case of the cationic liposome-mediated delivery compared to the nanotube-mediated delivery in vivo.

\section{A}

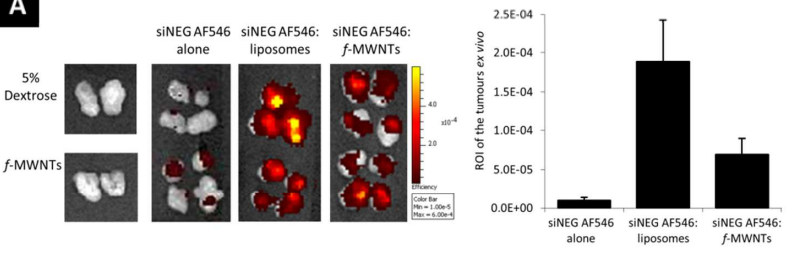

B

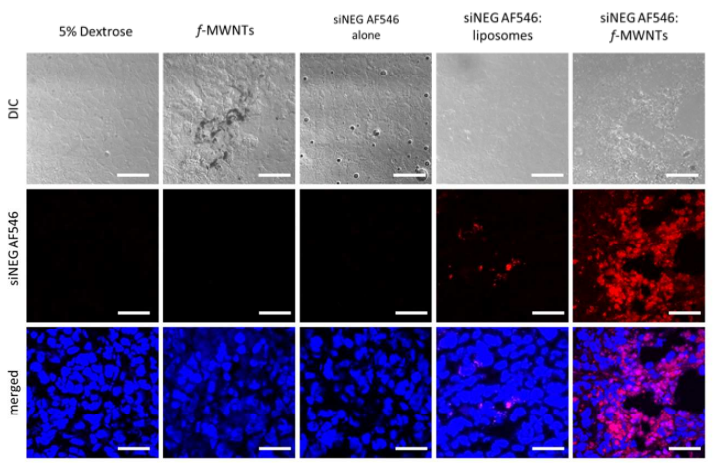

Figure 5: Uptake of siNEG AF546 in tumor xenografts. (A) Ex vivo imaging (left) and quantification of the fluorescence signals (right) detected from the dissected tumors by Xenogen IVIS imaging system. Tumors were dissected 24 hours after intratumoral administration of siNEG AF546 in free or complexed forms. (B) Intracellular uptake of siNEG AF546 in the dissected tumors by CLSM. Scale bars, $50 \mu \mathrm{m}$. 


\section{DISCUSSION}

We have previously reported the most comprehensive and promising study to date describing tumor apoptosis, delayed tumor growth and prolonged survival of human lung xenograft bearing mice after intratumoral administration of siRNA:f-MWNT complexes. ${ }^{22}$ In this previously reported study, we observed significant improvements in the therapeutic outcome in the group treated with siRNA:f-MWNT complexes compared to the lipoplexes treated group. That study utilized the proprietary sequence siTOX to induce tumor apoptosis and death, whilst ammonium functionalized MWNT and cationic liposomes were used as delivery vectors for the siRNA molecules. Although it was essential to investigate the reason behind the drastically improved performance of the ammonium functionalized MWNTs over cationic liposome vectors for siRNA delivery in vivo, due to the unknown nature of siTOX , it was difficult to perform a direct comparison between the two delivery systems at the molecular level (e.g. mRNA and protein synthesis level). Therefore, it was deemed essential to carry out further studies to correlate the pharmacokinetic profiles with bioactivity of the siRNA delivered by means of the two fundamentally different delivery systems. In this study the siTOX was substituted with a siRNA sequence specific for PLK1, a gene known to play an important role in cancer cell replication and proliferation, so successful gene silencing could lead to tumor growth arrest. Furthermore, we performed mechanistic studies substituting functional siRNA with fluorescently labeled non-coding SiRNA (siNEG) in order to determine the siRNA uptake into tumor mass in vivo and its retention in the tumor following intratumoral administration.

The main finding was that complexation of siPLK1 with MWNT- $\mathrm{NH}_{3}{ }^{+}$facilitated its internalization by tumor cells in solid tumor mass in vivo, resulting in significant PLK1 knockdown compared to the equivalent lipoplex formulation and all other treated groups. We didn't observe significant inhibition in tumor growth when treated with lipoplexes in our study although the lipoplexes worked in vitro (Fig. 1A-C). This could be due to the retention of lipoplexes into the tumor after intratumoral injection was not good and they were washed quickly while the CNT complexes were retained within the tumor so were longer acting, as observed from the 24 hours retention of fluorescence based siNEG AF546 in the free or complexed forms after intratumoral administration (Fig. 5B). Pharmacokinetic studies suggested that the bioavailability of siRNA within tumor cells after complexation with carbon nanotubes compared to the cationic liposomal formulation could be the rationale behind the superior bioactivity of the former delivery system. Whole tumor imaging by IVIS imaging system showed high florescence signals in tumors injected with the cationic liposomal formulation which was surprising and contradicting the bioactivity results. However, further inspection of tumor masses microscopically by CLSM confirmed that the majority of the siRNA was not delivered inside the cell and rather was retained in the interstitial space of the tumor in the case of the cationic liposome-based vectors. Non-internalized siRNA was most likely eliminated during the process of washing and fixation prior to imaging by CLSM, whilst internalized SiRNA was resilient to washing and was therefore visualized inside the cells by CLSM as was the case for $f$ MWNT-based carriers.

Cationic liposome based siRNA nanocarriers have been widely used for cancer therapy in different tumor models. These complexes were usually injected systemically ${ }^{28}$ that may explain the less therapy effects in our case. Previous studies have shown that liposomal systems exhibit broad interstitial diffusion within tumor mass after intratumoral injection, with slow clearance from the interstitial space. ${ }^{29}$ Prolonged accumulation of liposomes and the therapeutics within tumor mass does not always lead to enhanced intra-cellular delivery as it is necessary to establish if binding to the extra-cellular matrix does not hinder intra-cellular uptake into tumor cells. In conclusion of this 
study, carbon nanotube-mediated delivery exhibited the highest uptake in tumor cells as obtained by CLSM which conflicts with, conflicting the results obtained by whole body IVIS imaging which showed higher fluorescence signals of tumors injected with the lipoplexes. It is evident that detailed mechanistic studies utilizing multiple technique are necessary for are expressing the delivery efficiency of various delivery systems. Results obtained in this study demonstrated lack of toxicity of $f$-MWNTs and their efficiency as cytosolic siRNA carriers in addition to the bioactivity of the delivered siRNA as demonstrated by efficient gene silencing in vivo superseding the efficiency of cationic liposomal vectors as previously reported in other studies using stroke and cancer disease models. $^{15,22}$

Interestingly, amino functional groups on the MWNT- $\mathrm{NH}_{3}{ }^{+}$sidewalls can be further modified such as building dendritic structures as reported previously. ${ }^{26,27}$ Engineering the degree and type of dendritic branching is expected to affect the degree of siRNA association and gene silencing efficiency. We concluded from previous in vitro studies ${ }^{26,27}$ that increasing the degree of the dendritic branching on the $f$-MWNT side walls improved their water dispersibility, individualization and siRNA complexation. Future work should focus on comparing the bioactivity and pharmacokinetic profiles of other types of dendritic $f$-MWNT generations for siRNA delivery in vivo. In conclusion, we reformulated MWNT- $\mathrm{NH}_{3}{ }^{+}$by complexing with siPLK1 or siNEG AF546, to elucidate and understand of the efficient siRNA delivery in xenograft tumors. We proved that cationic multi-walled carbon nanotubes are efficient siRNA vectors for tumor eradication in vivo.

This work is a proof-of-principle study demonstrating that biocompatible $f$-MWNTs are effective intra-cellular transporters for siRNA in cancer gene therapy in vivo. So far, only a limited number of studies have reported $f$-CNTs as siRNA delivery systems for in vivo applications for diseases other than cancer. Lanner et al. presented successful CNT-based transfection of TRPC3 siRNA in adult skeletal muscle cells. The TRPC3 protein expression was knocked down, with functional effects seen in the inhibition of DAG-induced $\mathrm{Ca}^{2+}$ and decreases in the insulin-mediated glucose uptake..$^{30}$ This validated TRP 3 as a target for treatment of insulin-resistant conditions. Moreover, evidence has also shown that $f$-CNTs can effectively aid the transfection of generally difficult to transfect cells help the gene delivery, such as $\mathrm{T}$ cells and primary cells, including neurons and cardiomyocytes. ${ }^{31-34}$ Very recently, we reported MWNT- $\mathrm{NH}_{3}{ }^{+}$as transporters for Caspase-3 siRNA for the prevention of apoptosis following stroke induction in a murine Endothelin-1 stroke model. Functional recovery was achieved to peri-lesional levels after stereotactic administration of the therapy in both pre- and post-lesioned animals. ${ }^{15}$ Overall, although $f$-CNT-mediated gene silencing is at an early pre-clinical stage, recent studies have shown that $f$-CNT offer significant benefits in the area of gene therapy and more specifically gene silencing. 


\section{Experimental}

Materials. MWNTs were purchased from Nanostructured \& Amorphous Materials Inc., Huston, TX; Stock \#: $1240 \mathrm{XH}, 95 \%$, OD $20-30 \mathrm{~nm}$. MWNT- $\mathrm{NH}_{3}{ }^{+}$were prepared as previously described. ${ }^{27}$ Chemicals and solvents were obtained from Sigma-Aldrich (USA) and they were used as received. Liposomes were prepared by lipid film hydration, followed by filtration. Briefly, DOTAP:cholesterol ( $2: 1$ molar ratio) was dissolved in chloroform:methanol $(4: 1 \mathrm{v} / \mathrm{v})$, the organic solvent was evaporated in a rotary evaporator (Buchi, Switzerland) under vacuum at $40^{\circ} \mathrm{C}$ for $30 \mathrm{~min}$ and then flushed with a $\mathrm{N}_{2}$ stream to remove any residual traces of organic solvent. The dried lipid film was hydrated with $1 \mathrm{~mL}$ of $5 \%$ dextrose, sonicated and extruded twice through $0.1 \mu \mathrm{m}$ filter under sterile conditions. The final lipid concentration was $2 \mathrm{mM}$. AlexaFluor 546-labelled siNEG (siNEG AF546) (5' -> 3': UGCGCUACGAUCGACGAUG) and siPLK1 (5' -> 3': CCUUGAUGAAGAAGAUCAC) were purchased from Eurogentec (UK). Advanced RPMI, fetal bovine serum (FBS), penicillin/streptomycin, and phosphate buffered saline (PBS) were from Gibco, Invitrogen (UK). Human lung carcinoma Calu6 (HTB-55) were from ATCC (UK). BCA Protein assay kit, paraformaldehyde (PFA), Restore Plus Western Blot Stripping Buffer and ECL detection system were purchased from Thermo Scientific Ltd (USA). Mouse monoclonal GAPDH antibody were obtained from Ambion (Austin, USA) and HRP-linked anti-mouse IgG was obtained from Jackson ImmunoResearch (West Grove, MA, USA). Mouse monoclonal PLK1 antibody was purchased from Abcam (Cambridge, UK) and HRP-linked anti-rabbit IgG was obtained from Cell Signaling (MA, USA). Vectashield mounting media with DAPI (H1200) was purchased from Vector Laboratories (USA). Hybond ECL nitrocellulose membranes were obtained from GE Healthcare (UK). Complete Protease Inhibitor cocktail is from Roche. Unless otherwise stated, all others reagents were obtained from Sigma.

Liposome: siRNA and siRNA:MWNT-NH ${ }_{3}^{+}$complexation for biological studies. siRNA complex preparations for in vivo experiments were carried out by diluting the appropriate volume of liposome (1.4 preparations for in vivo experiments were carried out by diluting the appropriate volume of liposome (1.4
$\mathrm{mg} / \mathrm{mL})$ or $\mathrm{MWNT}-\mathrm{NH}_{3}{ }^{+}(1.28 \mathrm{mg} / \mathrm{mL})$ dispersion to a total volume of $25 \mu \mathrm{L}$ in $5 \%$ dextrosedextrose, to achieve siRNA;liposomes $\mathrm{N} / \mathrm{P}=4$ orsiRNA:MWNT- $\mathrm{NH}_{3}{ }^{+}$mass ratio $=8$. siRNA initial stock was prepared at $20 \mu \mathrm{M}$. An equal volume of $160 \mu \mathrm{g} / \mathrm{mL}$ siRNA in $5 \%$ dextrose was then added to the liposome or MWNT$\mathrm{NH}_{3}^{+}$aliquots and mixed by rapid pipetting, yielding a final siRNA concentration of $80 \mu \mathrm{g} / \mathrm{mL}$. Fifty $\mu \mathrm{L}$ of the complexes were injected per animal. A similar procedure was used to prepare complexes for in vitro experiments except that complexation was carried out initially in a total volume of $100 \mu \mathrm{L}$ then diluted $5 \mathrm{X}$ with experiments except that complexation was carried out initially in
serum-free media yielding siRNA final concentration of $100 \mathrm{nM}$.

In vitro PLK1 gene silencing in Calu6 cells and in vivo PLK1 gene silencing in Calu6 xenograft tumors. Calu6 cells $(30,000$ cells / well) were seeded in 6 -well plates. 24 hours later, cells were transfected with siPLK1 in different formulations. After 6hours, 24 hours or 48 hours post-transfection, cells were washed twice with ice-cold PBS before lysis. Tumor tissues were harvested and snap-frozen immediately in liquidnitrogen-cooled isopentane. Samples were stored at $-80^{\circ} \mathrm{C}$ prior to lysis. The tumors were placed in liquid nitrogen and ground thoroughly with a pestle. Decanted tissue powder was weighed in Eppendorfs before lysis.

Gene silencing by Western blot. Cells or decanted tissue powder were collected directly into lysis buffer (50 mM Tris-Cl, pH 8.0, $150 \mathrm{mM} \mathrm{NaCl}, 0.1 \%$ SDS, $1 \%$ Nonidet P-40 and $0.5 \%$ sodium deoxycholate). The tumor tissue lysate was sonicated and then left on ice for $30 \mathrm{~min}$. Samples were cleared by centrifugation at $15000 \mathrm{rpm}$ for $15 \mathrm{~min}$ at $4{ }^{\circ} \mathrm{C}$ and the supernatant was collected. Total protein concentration was assessed using BCA Protein assay kit and $10 \mu \mathrm{g}$ of total protein was taken from each supernatant and resolved on $10 \%$ SDS-PAGE gels and transferred to Hybond ECL nitrocellulose membranes. After blocking in $3 \% \mathrm{BSA}$ for overnight, the blots were incubated with rabbit monoclonal PLK1 antibody at 1:1000 dilution, followed by horseradish peroxidise (HRP)-linked anti-mouse IgG at 1:10000 dilution used as secondary antibody. The specific bands were detected with enhanced chemiluminescence $(E C L)$ detection system. Band intensities of the PLK1 were quantified by Image J. GAPDH was used as an internal reference (house-keeping) gene. Membranes were stripped, blocked and rehybridized with mouse anti-GAPDH antibody (1:4000 dilution), followed by incubation with HRP-conjugated antibody.

Gene silencing by real-time PCR Analysis. Total RNA was extracted with an RNeasy Mini Kit (QIAGEN) according to the manufacturer's instructions. The concentration of total RNA was determined by measuring the optical density at $260 \mathrm{~nm}$ and the purity was checked as the $260 \mathrm{~nm} / 280 \mathrm{~nm}$ ratio with expected values between 1.8 and 2.0. First strand cDNA was prepared from $1 \mu \mathrm{g}$ RNA in a total volume of $20 \mu \mathrm{L}$ using the between 1.8 and 2.0. First strand cDNA was prepared from $1 \mu \mathrm{g}$ RNA in a total volume of $20 \mu \mathrm{L}$ using the
QuantiTect Reverse Transcription Kit (QIAGEN). Real-time PCR was performed using the CFX96 ${ }^{\mathrm{TM}}$ RealTime PCR Detection System (BioRad). The reactions contained 1 X Fast SYBR ${ }^{\circledR}$ Green Master Mix (BioRad), each primer (h-PLK1 sense 5' ACA GTA TTC CCA AGC ACA TC3 '; h-PLK1 antisense 5' AAT GGT CAG GCA GGT GAT 3'; h-GAPDH sense 5' GGT CGG AGT CAA CGG ATT 3'; h-GAPDH antisense 5' ATC GCC 
CCA CTT GAT TTTG 3 ) at $200 \mathrm{nM}$ and $1 \mu \mathrm{L}$ of CDNA from reverse transcription PCR in a $25 \mu \mathrm{L}$ reaction. After an initial denaturation step at $95^{\circ} \mathrm{C}$ for $10 \mathrm{~min}$, amplification was carried out with 40 cycles of denaturation at $95^{\circ} \mathrm{C}$ for $10 \mathrm{sec}$ and annealing at $60^{\circ} \mathrm{C}$ for $30 \mathrm{sec}$. Amplification was followed by a melting curve analysis to confirm PCR product specificity. No signals were detected in no-template controls. All samples were run in triplicates and the mean value of each triplicate was used for further calculations. Relative gene expressions were calculated using the $\Delta \Delta C_{T}$ method. The quantity of GAPDH (housekeeping) transcript in each sample was used to normalize the amount of PLK1 transcripts, then the normalized value was compared to the normalized expression in naïve cells to calculate a fold change value.

Tumor xenograft animal model and survival studies. All animal experiments were performed in compliance with the UK Home Office Code of Practice for the Housing and Care of Animals Used in Scientific Procedures. Six to eight-weeks old female Swiss nude mice (Charles River Laboratories, UK) were caged in individually vented cages (IVC; Allentown, USA) in groups of four with free access to food and water. A temperature of $19-22^{\circ} \mathrm{C}$ was maintained, with a relative humidity of $45-65 \%$, and a 12 hours light/dark cycle. An established human lung Calu6 xenograft tumor model was applied as described previously. ${ }^{22}$ Mice were inoculated subcutaneously with $1 \times 10^{6}$ Calu6 human epithelial lung carcinoma cells mixed 1:1 with Matrigel (Becton Dickinson, UK) in $100 \mu \mathrm{L}$ on the both flanks subcutaneously. Intratumoral injections were performed when the tumor volume reached $0.2-0.4 \mathrm{~cm}^{3}$ and were carried out on days 17,20 , 2327 and 30 post-tumor implantation in this study. For intratumoral administration and tissue analysis, mice were anesthetized using isofluorane and injected with the siRNA alone or the complexed prepared in $5 \%$ dextrose. The needle was inserted in the longitudinal direction from the tumor edge into the center of the tumor, $50 \mu \mathrm{L}$ of the dispersion was administered slowly over 1 minute, and the needle was left in the tumor for another 5 minutes to prevent sample leakage.

Tumor growth was monitored by measuring the tumor size three times per week with Vernier calipers over a period of 30 days. The tumor volume was estimated by bilateral Vernier caliper measurement three per week and calculated using the formula (width $\mathrm{x}$ width) $\mathrm{x}$ (length) $\times(\pi / 6)$, where length was taken to be the longest diameter across the tumor. Implantation with $5 \%$ dextrose (naïve), carbon nanotubes alone and siRNA in different formulations were carried out twice weekly for all groups. Quadrupling time is used to compare the efficiency of different types of therapies used and is defined as the time required for a tumor to reach four efficiency of different types of therapies used and is defined as the time required for a tumor to reach four
times of its initial volume at the start of the therapy. ${ }^{35,36}$ Mice were sacrificed by cervical dislocation when tumor area reached $800-1000 \mathrm{~mm}^{3}$.

Neutral red and Heamatoxylin/Eosin (H\&E) tissue histology. For histological analysis, tumors samples and the major organs (heart, lung, liver, spleen and kidneys) were fixed in $10 \%$ buffered formalin and processed for routine histology with neutral red and hematoxylin and eosin (H\&E) stain by the Laboratory Drocessed for routine histology with neutral red and hematoxylin and eosin (H\&E) stain by the Laboratory carried out with Nikon Microphot-FXA microscope coupled with Infinity 2 digital camera.

TUNEL labeling. Tissue sections were deparaffinized in Histoclear and rehydrated through graded ethanol. The DeadEnd ${ }^{\mathrm{TM}}$ Fluorometric TUNEL System (Promega, UK) was used to label nicked DNA through incorporation of fluorescein-12-dUTP. Samples were incubated with recombinant Terminal Deoxynucleotidyl Transferase (rTdT) as per manufacturer's instructions and fluorescein labeling was visualized. Slices were visualized under $10 X$ lens using CLSM. DAPI was used to counterstain nuclei. DAPI and fluorescein were excited at $4 \%$ of 365 and $2 \%$ of 488 laser power, respectively.

In vivo and ex vivo optical imaging study. Optical imaging studies were performed using an IVIS spectrum small-animal in vivo imaging system (Caliper Lifescience, Hopkinton, MA). Mice bearing Calu6 tumors were intratumourallyintratumoral injected when the tumors reached $500 \mathrm{~mm}^{3}$, and imaged at 5 minutes, 15 minutesmin, 15 min, 30 minutes, 1 hourmin, 1 h, 2 hours, 3 hours, 4 hours and 24 hours postadministration. Isofluorane $(2 \%)$ was used for anesthesia during imaging. The fluorescence images were obtained with the following settings: exposure time (1 s), f/stop (2s), binning (4s) and field of view (24 s). Excitation filters were $535 \mathrm{~nm}$. All images were background subtracted using Living Image software. Tumors and different organs were dissected 24 hours post-injection. Tumors were cross-sectioned with the inside above and imaged immediately. The mean fluorescence intensity of the tumors was calculated using Living Image software.

Tissue analysis. 24 hours post-injection of siNEG AF546 in different formulations, the mice were killed and tumors samples were collected. Samples were stored at $-80^{\circ} \mathrm{C}$ prior to frozen sectioning. Frozen tumors were embedded into OCT and plunged into a liquid-nitrogen bath for at least 30 seconds. Samples were retrieved from the bath then sectioned using the cryostat at $-18^{\circ} \mathrm{C}$ into $10 \mu \mathrm{m}$-thick sections. The sections were mounted on a superfrost slide and left to dry at room temperature for 15-30 minutes. For tumor 
visualization, the sections were fixed for 3 minutes in cold acetone at $-20^{\circ} \mathrm{C}$, rinsed with PBS for 15 minutes at room temperature and mounted with Vectashield as a mounting medium. Nuclei were Nucleus

counterstained with DAPI. The slices were visualized under 40X lens using CLSM (LSM 710, Zeiss UK).

DAPI and AlexaFluor 546 were excited at $4 \%$ of 365 and $5 \%$ of 514 laser power, respectively. 


\section{Acknowledgements}

The work has been supported partially by the School of Pharmacy, University of London, the University of Trieste, Italian Ministry of Education MIUR (cofin Prot. 20085M27SS and firb prot. RBAP11ETKA) and Regione Friuli Venezia Giulia, the Centre National de la Recherche Scientifique (CNRS) and the European Union FP7 ANTICARB (FP7-HEALTH-2007-2.4.1-7) research programme. M.P. acknowledges financial support from ERC Advanced Grant Carbonanobridge (ERC-2008-AdG-227135).

\section{Supporting Information description}

Table of contents

\begin{tabular}{|l|l|}
\hline Supplementary Figure 1 & $\begin{array}{l}\text { Chemical structures and TEM images of positively charged } f \text {-MWNT } \\
\left(\mathrm{MWNT}-\mathrm{NH}_{3}^{+}\right) \text {. }\end{array}$ \\
\hline Supplementary Figure 2 & Histological examination of the major organs in tumor-bearing mice. \\
\hline Supplementary Figure 3 & $\begin{array}{l}\text { Quantification of the fluorescence signals detected in the tumor region } \\
\text { in xenograft-bearing mice after intratumoral administration. }\end{array}$ \\
\hline
\end{tabular}

\section{Keywords}

Gene silencing; polo-like kinase (PLK1); siRNA; xenograft; lung cancer; carbon nanotubes (CNTs) 


\section{REFERENCES}

lannazzo, D., Piperno, A., Pistone, A., Grassi, G., and Galvagno, S. (2013) Recent advances in carbon nanotubes as delivery systems for anticancer drugs. Current medicinal chemistry 20, 1333-54. Koff, J. L., Ramachandiran, S., and Bernal-Mizrachi, L. (2015) A Time to Kill: Targeting Apoptosis in Cancer. International journal of molecular sciences 16, 2942-2955.

3 Bremer, E., van Dam, G., Kroesen, B. J., de Leij, L., and Helfrich, W. (2006) Targeted induction of apoptosis for cancer therapy: current progress and prospects. Trends in molecular medicine 12, 382-93.

4 Zhang, H., Shi, X., Paddon, H., Hampong, M., Dai, W., and Pelech, S. (2004) B23/nucleophosmin serine 4 phosphorylation mediates mitotic functions of polo-like kinase 1. The Journal of biological chemistry 279, 35726-34. Cheng, L., Wang, C., and Jing, J. (2014) Polo-Like Kinase 1 as a Potential Therapeutic Target for Osteosarcoma. Current pharmaceutical design.

6 Golsteyn, R. M., Schultz, S. J., Bartek, J., Ziemiecki, A., Ried, T., and Nigg, E. A. (1994) Cell cycle analysis and chromosomal localization of human Plk1, a putative homologue of the mitotic kinases Drosophila polo and chromosomal localization of human Plk1, a putative homologue of

7 Holtrich, U., Wolf, G., Brauninger, A., Karn, T., Bohme, B., Rubsamen-Waigmann, H., and Strebhardt, K. (1994) Holtrich, U., Wolf, G., Brauninger, A., Karn, T., Bohme, B., Rubsamen-Waigmann, H., and Strebhardt, K. (1994)
Induction and down-regulation of PLK, a human serine/threonine kinase expressed in proliferating cells and tumors. Induction and down-regulation of PLK,
Proc Natl Acad Sci U S A 91, 1736-40.

8 Groc Natl Acad Sci U S A 91, 1736-40. Garland, L. L., Taylor, C., Pilkington, D. L., Cohen, J. L., and Von Hoff, D. D. (2006) A phase I pharmacokinetic
study of HMN-214, a novel oral stilbene derivative with polo-like kinase-1-interacting properties, in patients with advanced solid tumors. Clin Cancer Res 12, 5182-9.

9 Ahmad, N. (2004) Polo-like kinase (Plk) 1: a novel target for the treatment of prostate cancer. FASEB J 18, 5-7. Reagan-Shaw, S., and Ahmad, N. (2005) Polo-like kinase (Plk) 1 as a target for prostate cancer management. lubmb Life 57, 677-682.

Spankuch-Schmitt, B., Bereiter-Hahn, J., Kaufmann, M., and Strebhardt, K. (2002) Effect of RNA silencing of pololike kinase-1 (PLK1) on apoptosis and spindle formation in human cancer cells. J Natl Cancer Inst 94, 1863-77. Spankuch-Schmitt, B., Wolf, G., Solbach, C., Loibl, S., Knecht, R., Stegmuller, M., von Minckwitz, G., Kaufmann, M., and Strebhardt, K. (2002) Downregulation of human polo-like kinase activity by antisense oligonucleotides induces growth inhibition in cancer cells. Oncogene 21, 3162-71.

$13 \mathrm{Hu}, \mathrm{K}$., Law, J. H., Fotovati, A., and Dunn, S. E. (2012) Small interfering RNA library screen identified polo-like kinase-1 (PLK1) as a potential therapeutic target for breast cancer that uniquely eliminates tumor-initiating cells Breast cancer research : BCR 14, R22.

14 Maire, V., Nemati, F., Richardson, M., Vincent-Salomon, A., Tesson, B., Rigaill, G., Gravier, E., Marty-Prouvost, B., De Koning, L., Lang, G., et al. (2013) Polo-like kinase 1: a potential therapeutic option in combination with conventional chemotherapy for the management of patients with triple-negative breast cancer. Cancer research 73 , 813-23.

15 Al-Jamal, K. T., Gherardini, L., Bardi, G., Nunes, A., Guo, C., Bussy, C., Herrero, M. A., Bianco, A., Prato, M., Kostarelos, K., et al. (2011) Functional motor recovery from brain ischemic insult by carbon nanotube-mediated siRNA silencing. Proceedings of the National Academy of Sciences of the United States of America 108, 10952-7. Kostarelos, K., Bianco, A., and Prato, M. (2009) Promises, facts and challenges for carbon nanotubes in imaging and therapeutics. Nat Nanotechnol 4, 627-33.

17 Kam, N. W., Liu, Z., and Dai, H. (2005) Functionalization of carbon nanotubes via cleavable disulfide bonds for

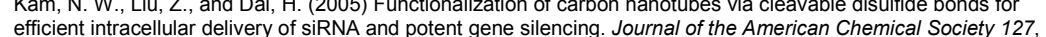
12492-3.

18 Zhang, Z., Yang, X., Zhang, Y., Zeng, B., Wang, S., Zhu, T., Roden, R. B., Chen, Y., and Yang, R. (2006) Delivery of telomerase reverse transcriptase small interfering RNA in complex with positively charged single-walled carbon nanotubes suppresses tumor growth. Clinical cancer research : an official journal of the American Association for

Cancer Research 12, 4933-9.
Wang, X., Ren, J., and Qu, X. (2008) Targeted RNA interference of cyclin A2 mediated by functionalized singlewalled carbon nanotubes induces proliferation arrest and apoptosis in chronic myelogenous leukemia K562 cells. ChemMedChem 3, 940-5

Chen, H., Ma, X., Li, Z., Shi, Q., Zheng, W., Liu, Y., and Wang, P. (2012) Functionalization of single-walled carbon nanotubes enables efficient intracellular delivery of siRNA targeting MDM2 to inhibit breast cancer cells growth. Biomedicine \& pharmacotherapy = Biomedecine \& pharmacotherapie 66, 334-8.

Wang, L., Shi, J., Zhang, H., Li, H., Gao, Y., Wang, Z., Wang, H., Li, L., Zhang, C., Chen, C., et al. (2013) Synergistic anticancer effect of RNAi and photothermal therapy mediated by functionalized single-walled carbon nanotubes. Biomaterials 34, 262-74.

22 Podesta, J. E., Al-Jamal, K. T., Herrero, M. A., Tian, B., Ali-Boucetta, H., Hegde, V., Bianco, A., Prato, M., and Kostarelos, K. (2009) Antitumor activity and prolonged survival by carbon-nanotube-mediated therapeutic siRNA silencing in a human lung xenograft model. Small 5, 1176-85

23 Al-Jamal, K. T., Toma, F. M., Yilmazer, A., Ali-Boucetta, H., Nunes, A., Herrero, M. A., Tian, B., Eddaoudi, A., AlJamal, W. T. Bianco, A et al. (2010) Enhanced cellular internalization and gene silencing with a series of cationic dendron-multiwalled carbon nanotube:siRNA complexes. FASEB journal : official publication of the Federation of American Societies for Experimental Biology 24, 4354-65.

24 Battigelli, A., Wang, J. T., Russier, J., Da Ros, T., Kostarelos, K., Al-Jamal, K. T., Prato, M., and Bianco, A. (2013) Ammonium and guanidinium dendron-carbon nanotubes by amidation and click chemistry and their use for siRNA delivery. Small 9, 3610-9.

ACS Paragon Plus Environment 
25 Bartholomeusz, G., Cherukuri, P., Kingston, J., Cognet, L., Lemos, R., Leeuw, T. K., Gumbiner-Russo, L., Weisman, R. B., and Powis, G. (2009) In Vivo Therapeutic Silencing of Hypoxia-Inducible Factor 1 Alpha (HIF1alpha) Using Single-Walled Carbon Nanotubes Noncovalently Coated with siRNA. Nano Res 2, 279-291.

26 Al-Jamal, K. T., Toma, F. M., Yilmazer, A., Ali-Boucetta, H., Nunes, A., Herrero, M. A., Tian, B., Eddaoui, A., AlJamal, W. T., Bianco, A., et al. (2010) Enhanced cellular internalization and gene silencing with a series of cationic dendron-multiwalled carbon nanotube:siRNA complexes. FASEB J.

27 Herrero, M. A., Toma, F. M., Al-Jamal, K. T., Kostarelos, K., Bianco, A., Da Ros, T., Bano, F., Casalis, L., Scoles, G., and Prato, M. (2009) Synthesis and characterization of a carbon nanotube-dendron series for efficient siRNA delivery. Journal of the American Chemical Society 131, 9843-8.

28 Zimmermann, T. S., Lee, A. C., Akinc, A., Bramlage, B., Bumcrot, D., Fedoruk, M. N., Harborth, J., Heyes, J. A. Jeffs, L. B., John, M., et al. (2006) RNAi-mediated gene silencing in non-human primates. Nature 441, 111-4.

29 Bao, A. D., Phillips, W. T., Goins, B., Zheng, X. P., Sabour, S., Natarajan, M. Woolley, F. R., Zavaleta, C., and Otto, R. A. (2006) Potential use of drug carried-liposomes for cancer therapy via direct intratumoral injection. International Journal of Pharmaceutics 316, 162-169.

30 Lanner, J. T., Bruton, J. D. Assefaw-Redda, Y., Andronache, Z., Zhang, S. J., Severa, D., Zhang, Z. B., Melzer, W., Zhang, S. L., Katz, A., et al. (2009) Knockdown of TRPC3 with siRNA coupled to carbon nanotubes results in Zhang, S. L., Katz, A., et al. (2009) Knockdown of TRPC3 with siRNA coupled to carbon nanotubes results in decreased insulin-mediated glucose uptake in adult skeletal muscle cels.

31 Cai, D., Mataraza, J. M., Qin, Z. H., Huang, Z. P., Huang, J. Y., Chiles, T. C., Carnahan, D., Kempa, K., and Ren, Z F. (2005) Highly efficient molecular delivery into mammalian cells using carbon nanotube spearing. Nature Methods

Liu, Z., Winters, M., Holodniy, M., and Dai, H. (2007) siRNA delivery into human T cells and primary cells with carbon-nanotube transporters. Angew Chem Int Ed Engl 46, 2023-7.

Krajcik, R., Jung, A., Hirsch, A., Neuhuber, W., and Zolk, O. (2008) Functionalization of carbon nanotubes enables non-covalent binding and intracellular delivery of small interfering RNA for efficient knock-down of genes. Biochemical and biophysical research communications 369, 595-602.

Ladeira, M. S., Andrade, V. A., Gomes, E. R., Aguiar, C. J., Moraes, E. R., Soares, J. S., Silva, E. E., Lacerda, R. G., Ladeira, L. O., Jorio, A., et al. (2010) Highly efficient siRNA delivery system into human and murine cells using single-wall carbon nanotubes. Nanotechnology 21, 385101.

Kurizaki, T., Okazaki, S., Sanderson, S. D., Colcher, D., Enke, C. A., Tempero, M. A., and Baranowska-Kortylewicz, J. (2002) Potentiation of radioimmunotherapy with response-selective peptide agonist of human C5a. J Nucl Med $43,957-67$

$36 \mathrm{Wu}, \mathrm{J}$., and Houghton, P. J. (2009) Assessing cytotoxic treatment effects in preclinical tumor xenograft models. J Biopharm Stat 19, 755-62. 


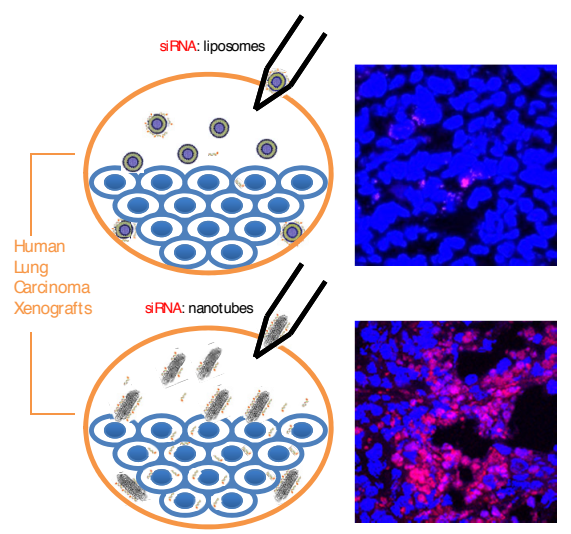

ACS Paragon Plus Environment 